\title{
Single-particle-ICP-MS advances
}

\author{
Antonio R. Montoro Bustos ${ }^{1}$ • Michael R. Winchester ${ }^{1}$
}

Published online: 21 May 2016

(C) Springer-Verlag Berlin Heidelberg (outside the USA) 2016

It has been said that you cannot teach an old dog new tricks. Inductively coupled plasma mass spectrometry (ICP-MS) has been around for several decades, with the first commercial ICP-MS instruments appearing more than 30 years ago. Some scientists might be a bit surprised that this mature old dog has any new tricks. Nevertheless, the implementation of ICP-MS instruments to perform measurements on individual nanoparticles, commonly called single-particle (sp)ICP-MS, is just this sort of new trick.

While spICP-MS was first reported in the scientific literature 13 years ago [1], it has only started to gain traction in the last few years. The interest in spICP-MS is exploding globally, as evidenced by examining the technical programs of relevant international conferences from the last several years. More and more laboratories are investing resources in developing and applying the technique, and ICP-MS instrument manufacturers are now, or soon will be, offering hardware modifications and dedicated software packages to enable easy spICPMS measurements.

Generally, spICP-MS is used to characterize populations of nanoparticles suspended in aqueous solutions. A very dilute suspension is introduced into the ICP-MS instrument, such that statistically only one nanoparticle at a time enters the plasma. The plasma atomizes and ionizes the constituents of the nanoparticle, which are then quantified using the mass spectrometer. The parameters of the nanoparticle population that can be measured include the mean size, size distribution,

Published in the topical collection Single-particle-ICP-MS Advances with guest editors Antonio R. Montoro Bustos and Michael R. Winchester.

Michael R. Winchester

mwinchester@nist.gov

1 Chemical Sciences Division, National Institute of Standards and Technology, Gaithersburg, MD 20899, USA nanoparticle number concentration, and nanoparticle mass concentration. Nanoparticle size and size distribution have received the most attention to date.

A strength of spICP-MS is that it can be performed using off-the-shelf ICP-MS instrumentation. Strictly speaking, no instrument modifications are required, although data reduction can be cumbersome. As spICP-MS becomes further developed, additional or uncommon instrument capabilities, such as very short detector dwell times (on the order of microseconds), automated data reduction software, and hyphenation with a front-end separation technique, such as field-flow fractionation, may begin to be viewed as essential. These are the types of improvements now being pursued by ICP-MS instrument manufacturers.

Another important strength of spICP-MS is its superb detection capability in terms of nanoparticle number (or mass) concentration. The technique can readily be used to detect and characterize nanoparticles at the extremely low number concentrations that are often found in real-world environmental samples (e.g., in the range $10^{3} \mathrm{~cm}^{-3}$ to $10^{5} \mathrm{~cm}^{-3}$ [2]). This is a concentration regime where competing techniques dare not tread. For example, even finding a nanoparticle in such an environmental sample using electron microscopy can be a challenge. Given the ongoing, worldwide, intense research interest in understanding the environmental, health, and safety (EHS) implications of engineered nanoparticles, this unique spICP-MS measurement capability should not be undervalued.

However, there are shortcomings of spICP-MS, as well. For example, the technique can measure only one, or at most two, isotopes in a single analysis using quadrupole instruments, the most common type of ICP-MS instrument. Also, while the detection power in terms of nanoparticle number concentration is exceptionally good, the detection power in terms of nanoparticle size is still somewhat lacking, with typical limits of detection (LODs), expressed as equivalent spherical diameter, ranging from $10 \mathrm{~nm}$ to $20 \mathrm{~nm}$ for monoisotopic 
nanoparticles. The size-based LODs become poorer when the nanoparticles are composed of more than one isotope.

Another shortcoming is the fact that real-world samples usually must be altered to be compatible with common ICPMS sample introduction systems. Partly for this reason, most studies and advancements in spICP-MS to date have been performed using pristine nanoparticles as test cases - nanoparticles and matrices that are simple from a physicochemical viewpoint. The necessity to modify a real sample prior to sample introduction tends to offset, to a degree, the advantage of the high detection power in terms of nanoparticle number concentration. Of course, the requirement to prepare samples for analysis is not unique to spICP-MS, but is true of all competing techniques, as well.

The above comments should make it clear that, while considerable progress has been made, spICP-MS is still best considered an emerging technique, with many advancements yet to come. Nevertheless, it would be wrong to conclude that spICP-MS cannot now be employed in the laboratory. In fact, the technique is already being applied as both a research tool and a metrological tool.

This issue of Analytical and Bioanalytical Chemistry contains a topical collection of papers focused on current developments and applications of spICP-MS. We would like to express our gratitude to all of the authors who submitted manuscripts to be considered for inclusion in this topical collection. We hope that the readers will find the collection educational and useful for their own endeavors.

\section{References}

1. Degueldre C, Favarger PY. Colloid analysis by single particle inductively coupled plasma mass spectrometry: a feasibility study. Colloid Surf A. 2003;217:137-42.

2. Pace HE, Rogers NJ, Jarolimek C, Coleman VA, Higgins CP, Ranville JF. Determining transport efficiency for the purpose of counting and sizing nanoparticles via single particle inductively coupled plasma mass spectrometry. Anal Chem. 2011;83:9361-9.

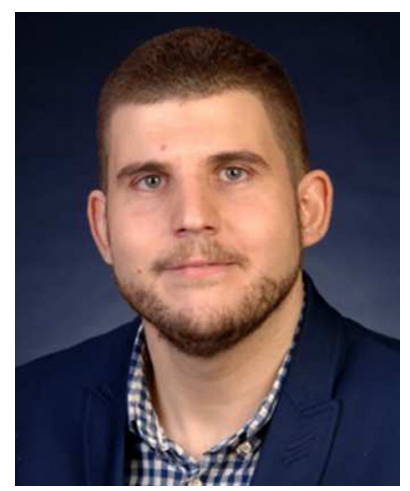

Antonio R. Montoro Bustos is a Guest Scientist at the National Institute of Standards and Technology (NIST) in Gaithersburg, Maryland (USA). He received his Ph.D. degree in Analytical Chemistry from The University of Oviedo (Spain) under the direction of Dr. Jorge Ruiz Encinar and Prof. Alfredo Sanz Medel. His research efforts are focused on the application of singleparticle ICP-MS for the characterization of nanomaterials at environmentally relevant concentrations, including the validation of the technique, the evaluation of the role of different coatings on the stability of nanoparticles and the study of matrix effects on spICP-MS analytical performance.

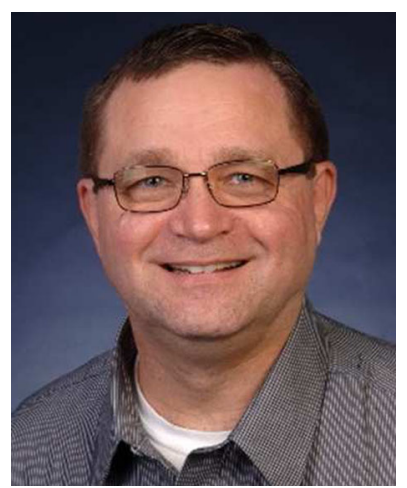

Michael R. Winchester is a Supervisory Research Chemist and Leader of the Inorganic Measurement Science Group in the Chemical Sciences Division of NIST in Gaithersburg, Maryland (USA). He began his career at NIST in 1991 as a National Research Council Postdoctoral Associate, immediately after earning the Ph.D. degree in Analytical Chemistry from Clemson University under the direction of Prof. R. Kenneth Marcus. Over the years, his research interests have involved glow discharge spectroscopy; Fourier transform spectroscopy in the ultraviolet/visible spectral range; ultralow bias, small uncertainty analysis using atomic spectroscopy; advancements in reference materials; and other topics. His most recent research interest is in the area of physicochemical characterizations of engineered nanomaterials. The NIST group that he leads has dedicated research programs in this area, as well as in seawater $\mathrm{pH}$ metrology and other areas, and is also responsible for hundreds of NIST reference materials. 